\title{
Ubiquidade e mobilidade de Objetos de Aprendizagem usando o papel como recurso
}

\author{
Núbia dos Santos Rosa Santana dos Santos, PGIE/UFRGS, nubrosa@ gmail.com \\ José Valdeni de Lima, PGIE/UFRGS, valdeni@inf.ufrgs.br \\ Leandro Krug Wives, Instituto de Informática/UFRGS, wives@inf.ufrgs.br
}

\begin{abstract}
Resumo. Atualmente, existem diferentes possibilidades de acessar conteúdos pedagógicos digitais, tais como Internet, dispositivos móveis e, inclusive, TV digital. Um problema relevante, no entanto, é que nem sempre os dispositivos estão disponíveis ao usuário (aluno). Nesse contexto, este artigo propõe o uso do papel como meio de aprendizagem ubíqua (integrado a estratégias pedagógicas), podendo ser utilizado ainda como mecanismo de interação ou entrada de dados para outros dispositivos. O artigo também apresenta uma sugestão de estratégia que pode ser usada em sala de aula e/ou no âmbito do elearning e m-learning.
\end{abstract}

Palavras-chaves: ubiquidade, mobilidade, objetos de aprendizagem

\section{Ubiquity and mobility of Learning Objects using the paper as a resource}

\begin{abstract}
Nowadays, there are different possibilities to access digital educational content, i.e., Internet, mobile, and digital TV. A relevant problem, however, is that devices are not always available to the user (student). In this context, this paper proposes the use of paper sheets as a way of ubiquitous learning (integrated to teaching strategies) and can also be used as an interaction mechanism or input for other devices. This paper also suggests a strategy that could be used in the classroom and/or in the e-learning and mlearning.
\end{abstract}

Keywords: ubiquity, mobility, learning objects

\section{1- Introdução}

Vários tipos de recursos de Tecnologia da Informação e Comunicação (TIC) podem ser utilizados como auxílio ao processo de ensino e aprendizagem, entre eles: o computador, vídeos, televisão, rádio, etc. Entre as TIC destacam-se também os Objetos de Aprendizagem (OA), os quais podem ser definidos como "qualquer entidade, digital ou não digital, que possa ser utilizada, reutilizada ou referenciada durante o aprendizado suportado por tecnologias" (IEEE, 2002). Considerando a existência de 
diferentes meios de acesso aos conteúdos digitais é fundamental que os recursos educacionais, como os OA, sejam ubíquos, multimodais e interativos.

Dessa forma, a ubiqüidade é um fator importante uma vez que existem várias possibilidades de acessar e usar conteúdos digitais em qualquer momento, independente de lugar. Os OA também devem ser multimodais (considerando a materialização do conteúdo), isto é, desenvolvidos para possibilitar a interação de vários modos. Com isso, um OA poderia ser acessado através da Internet, de dispositivos móveis e inclusive pela TV digital (TVD), oferecendo maior facilidade no uso de recursos educacionais.

Este artigo analisa a possibilidade da mídia impressa (papel) como um dos recursos que possibilita a ubiquidade e mobilidade para uso de recursos digitais. Mesmo apresentando limitações devido a sua característica estática, o papel pode ser utilizado como dispositivo de ligação para a mídia digital, inclusive com a TV digital (Gomes, 2009).

Sellen e Harper (2001) ressaltam quatro características-chave do papel, sendo elas: a possibilidade de navegação rápida e flexível através de um documento, a inserção de anotações durante a leitura, a mobilidade e a integração de atividades híbridas, tais como escrita e leitura.

A proposta deste artigo é a integração do papel, um dos recursos mais utilizados na educação, com recursos digitais através da tecnologia QR-Code. O QR-Code é uma matriz (código de barras bidimensional), criado em 1994 pela empresa japonesa DensoWave. O artigo apresenta também uma sugestão de estratégia usando o papel com QRCodes para acessar conteúdos interativos em Objetos de Aprendizagem.

Nesse contexto, o uso do papel pode ser aplicado para facilitar a mobilidade e a ubiquidade de conteúdos, podendo ser utilizado como dispositivo de entrada para interação com outros dispositivos.

A seção 2 apresenta alguns meios de acesso a conteúdos e trabalhos relacionados. A seção 3 apresenta uma proposta de uso do papel integrado à tecnologia do QR-Code e, ainda, propõe uma estratégia para uso educacional. Por fim, a seção 4 apresenta as considerações finais.

\section{2- Meios de acesso a conteúdos educacionais}

Os conteúdos educacionais podem ser acessados de diferentes maneiras: através da Web, de dispositivos móveis e TV digital, ou mesmo através de mídia impressa como mostram trabalhos já realizados (Gomes, 2009). Alguns conceitos como Mobile learning, ou m-learning, e t-learning estão relacionados à forma como os recursos são acessados. Segundo O’Malley (2005) o m-learning é qualquer tipo de aprendizado que acontece quando o aluno não está em um lugar fixo e predeterminado, ou, ainda, é a aprendizagem que ocorre quando o aluno aproveita as oportunidades de aprendizagem oferecidas pelas tecnologias móveis. Já o t-learning é considerado por Lytras et al. (2002) como a convergência de duas tecnologias distintas: televisão e computador.

A proposta deste artigo, no momento, tem como foco utilizar o papel como meio de acesso aos recursos do OA na Web através de dispositivos móveis (que possuem câmeras acopladas), mas pretende-se, posteriormente, usar o mesmo processo (com recursos do QR-Code) para acessar conteúdos educacionais na TV digital.

Conforme destacam Furlan e Ehrenberg (2009), a mobilidade característica do celular permitiu a comunicação entre pessoas a qualquer hora, o envio de simples mensagens ou completos e-mails para pessoas em trânsito e o acesso à internet sem fios 
(Wireless). E os aparelhos podem substituir vários aparelhos eletrônicos, como relógio, despertador, agenda, etc. $\mathrm{O}$ acúmulo das funções permite, através de apenas um aparelho, o celular, acessar e-mails, assistir TV, ouvir música, pagar contas, etc. Com a vantagem da mobilidade o celular passou a ser o foco de grandes empresas de tecnologia, e exemplo disso são os smartphones, que possibilitam a navegação na internet e sincronização de $e$-mails (Furlan e Ehrenberg, 2009).

Pellanda (2009) ressalta que, à medida que os dispositivos móveis começam a incorporar mais funcionalidades, eles se tornam mais parecidos com computadores e possuem grande relevância para no processo de inclusão digital, devido ao fato de serem mais baratos e possuírem a condição ubíqua. Além disso, Pellanda (2009) aborda a questão de que grande parcela da população não tem ainda acesso a telefones fixos, mas a tecnologia sem fio transpõe esse problema por não precisar de ligações diretas com as residências. Essa flexibilidade, aliada a uma expansibilidade, é um dos principais fatores de inclusão digital da tecnologia.

Além das possibilidades de acesso a conteúdos educacionais usando as tecnologias mencionadas acima, pode-se utilizar também o papel como meio para entrada de dados e interação com outros dispositivos. Alguns trabalhos relacionados à proposta deste artigo abordam o uso de papel como dispositivo de entrada de dados e o uso de etiquetas contendo informações para acesso a diferentes recursos. Gomes (2009) apresenta uma proposta que consiste de material didático impresso com links (códigos de barras) para objetos de aprendizagem a serem visualizados e/ou manipulados na TVD.

Klemmer et al. (2003), citam categorias de tecnologias que são apropriadas para reconhecer o papel como dispositivo de entrada de dados. São elas:

- etiquetas eletrônicas passivas (e.g., etiquetas RFID - Radio Frequency IDentification e IR - Infra-Red);

- etiquetas eletrônicas ativas (e.g., motes);

- etiquetas visuais (inclui 2D, glyphs, QR-codes);

- análise de conteúdo baseada no processamento de imagens (e.g., OCR Optical Character Recognition)

Entre as possibilidades de tecnologias apropriadas para que o papel funcione como dispositivo de entrada, este artigo destaca o uso do QR-Code. Miyaoku, Tang e Fels (2007) relatam que códigos como o QR-Code permitem uma quantidade de dados de aplicação prática, como uma URL, e o custo computacional para decodificação é muito baixo, podendo ser usados em dispositivos móveis, tais como telefones celulares.

Al-Khalifa (2008) apresenta uma proposta de uso do QR-Code para a identificação de objetos em um ambiente para deficientes visuais e cegos através de interação em tempo real. O sistema é baseado na utilização do QR-Code e através de uma câmera digital equipada com software leitor de QR-Code, o leitor decodifica o código em um endereço URL, e direciona para um arquivo de áudio na Web com a descrição verbal do objeto. O autor ressalta duas características importantes encontradas nos dispositivos modernos: a integração de câmeras digitais e a capacidade de acessar a Internet a qualquer hora e em qualquer lugar permitindo procurar informações quando precisamos.

Ghiron, Medaglia e Perrone (2009) apresentam a proposta do projeto Artsonomy, que permite aos usuários associar palavras-chave (tags) para obras de arte (por exemplo, em um museu) marcadas com um QR-Code, por meio de um aplicativo 
em um dispositivo móvel. As tags do usuário são coletadas no site Artsonomy permitindo assim observar e compartilhar com outros a experiência depois de ver uma obra de arte.

Yoshida, Miyaoku e Satou (2007) apresentam o "Mobile Magic Hand" um sistema de interface baseado em código. Com ele o usuário pode manipular os objetos virtuais sem precisar manter a câmera centrada no código visual, pois o sistema percebe bem as principais funções de rotação, inclinação e distância. Um exemplo de aplicação constitui-se em um jogo que permite manipular objetos virtuais impressos em um pedaço de papel, fazendo gestos naturais. Nesse caso, o usuário pode abrir a porta por inclinação, destrancar o cofre por rotação, e abrir uma gaveta fazendo um apontamento.

Ramsden (2008) apresenta algumas sugestões de uso do QR-Code como, por exemplo, apresentações durante uma aula. Outro exemplo citado pelo autor é o uso para respostas fechadas ( $\operatorname{sim} /$ não) onde o usuário pode escolher o QR-Code correspondente a opção desejada e enviar por SMS, com a vantagem de preservar o anonimato durante uma reunião. Tanaka e Teshigawara (2007) propõem um método de autenticação que utiliza um telefone celular e um leitor de código, evitando o envio de informações de autenticação entre um terminal de usuário e um prestador de serviços de rede através da Internet.

O QR-Code também tem sido utilizado em livros. Um exemplo prático de seu uso é o livro "Around the world in 80 days" (Verne, 2010). Com um dispositivo móvel é possível participar de conversas on-line sobre o livro, seus personagens ou autor. $\mathrm{O}$ livro possui versões em áudio e vídeo, que podem ser transmitidas ou descarregadas para o celular.

Alguns trabalhos mencionados nessa seção utilizam a tecnologia QR-Code e apresentam algumas possibilidades para o uso desta tecnologia também na elaboração de conteúdo educacional. A seção a seguir apresenta uma proposta de uso de QR-Code para a interação com um OA usando o papel.

\section{3- O uso do papel integrado à tecnologia QR-Code}

Segundo Heath e Luff (2000), o papel possibilita flexibilidade de interação para a colaboração síncrona e assíncrona, e permite aos indivíduos uma variedade de maneiras de participação em tarefas de interação. Dessa forma, pode-se utilizar o papel como dispositivo de entrada de dados e, com isso, propiciar maior mobilidade ao material educacional e a interatividade através do uso de outras mídias.

Neste trabalho são utilizadas as etiquetas visuais representadas por QR-Codes. Considerando todos os recursos presentes em um Objeto de Aprendizagem, como textos, imagens, áudios, vídeos, etc., tais recursos podem ser disponibilizados de diferentes formas. Um Objeto de Aprendizagem, planejado considerando a mídia impressa, pode conter elementos textuais e etiquetas que direcionem para os elementos dinâmicos. Nesse caso, o aluno poderia visualizar o conteúdo textual de um Objeto de Aprendizagem no papel e ao mesmo tempo ter a possibilidade de acessar todo o conteúdo multimídia do mesmo OA através dos links disponíveis nas etiquetas.

Para a visualização de uma animação, vídeo, áudio, conteúdo textual ou conteúdo extra (e.g., página web) referente a um OA, o aluno poderia utilizar o QRCode. Nesse caso, o aluno usando um celular com câmera e acesso à Internet, conseguiria acessar qualquer recurso dinâmico relacionado ao OA. A Figura 1 mostra o 
processo de acesso a um OA ou a conteúdos dinâmicos do mesmo usando um dispositivo móvel e o papel.

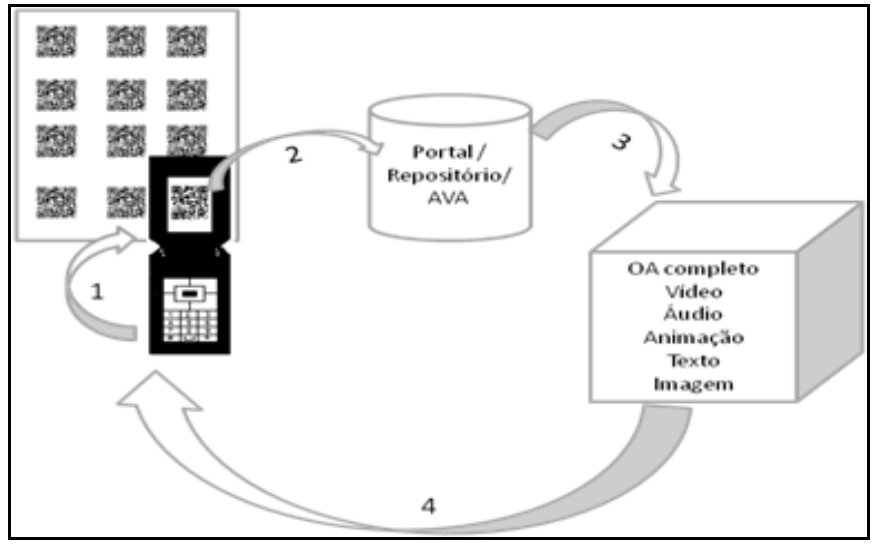

Figura 1 - Processo de acesso a OA (e conteúdos dinâmicos de um OA) através de papel e QR-Code

Para gerar o QR-Code, existem alguns geradores disponíveis na Internet, tais como Quickmark, i-nigma e kaywa. Para decodificar o QR-Code, o celular precisa possuir um leitor. Os celulares mais modernos já vêm com o leitor instalado, mas para os celulares que não possuem o leitor existe a possibilidade de fazer o download de um leitor como, por exemplo, o i-nigma e Kaywa (que também são geradores de QR-Code). A Figura 2 apresenta o processo de codificação e decodificação da informação contida em um QR-Code.

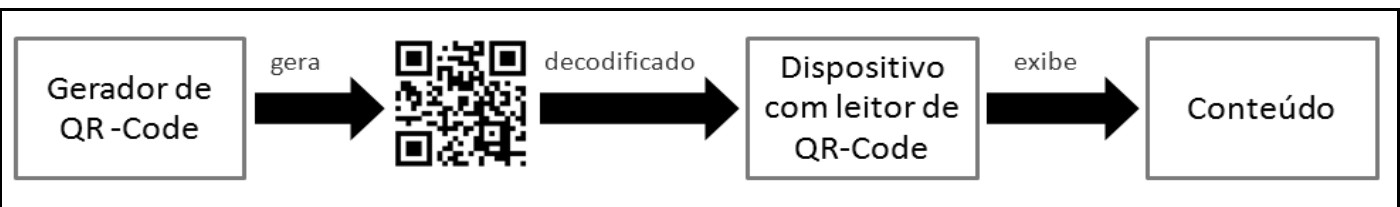

Figura 2. Codificação e decodificação da informação

A subseção seguinte apresenta uma estratégia de exploração de um OA, em desenvolvimento, usando o papel com QR-Code e um dispositivo móvel.

\section{1- Estratégias para uso integrado de papel e mídias}

O OA em desenvolvimento, Balança (Figura 3), é da área de matemática, mais especificamente sobre grandezas proporcionais, conteúdo apresentado na $6^{\mathrm{a}}$ série do ensino fundamental. Baseando-se no OA Gangorra Interativa (Melo et al., 2008) o OA Balança está sendo desenvolvido usando Flash e ActionScript. Conforme descrito em Melo et al. (2008), o Gangorra Interativa é um OA que faz a simulação de uma gangorra de pesos como as usadas por crianças em parques de diversão, e o objetivo é que os pesos em cada lado da gangorra sejam equilibrados.

$\mathrm{Na}$ elaboração do OA estão sendo consideradas as recomendações de usabilidade, apresentadas por Barbosa, Roesler e Reategui (2009), como cor de fundo, cor de fonte, entre outras, apropriadas para conteúdos acessados pela $\mathrm{Web}$, dispositivos 
móveis e TVD. No momento, o OA em desenvolvimento funciona na Web e em dispositivos móveis, e está sendo testado. Posteriormente, algumas alterações serão realizadas de forma a refinar sua interface.

Relacionando a teoria da aprendizagem significativa de Ausubel (2003), ela envolve uma interação seletiva entre o novo material de aprendizagem e as idéias preexistentes na estrutura cognitiva. Nesse sentido, um OA pode ser utilizado como organizador prévio, servindo como ponte entre o conhecimento já existente e o conhecimento a ser adquirido.

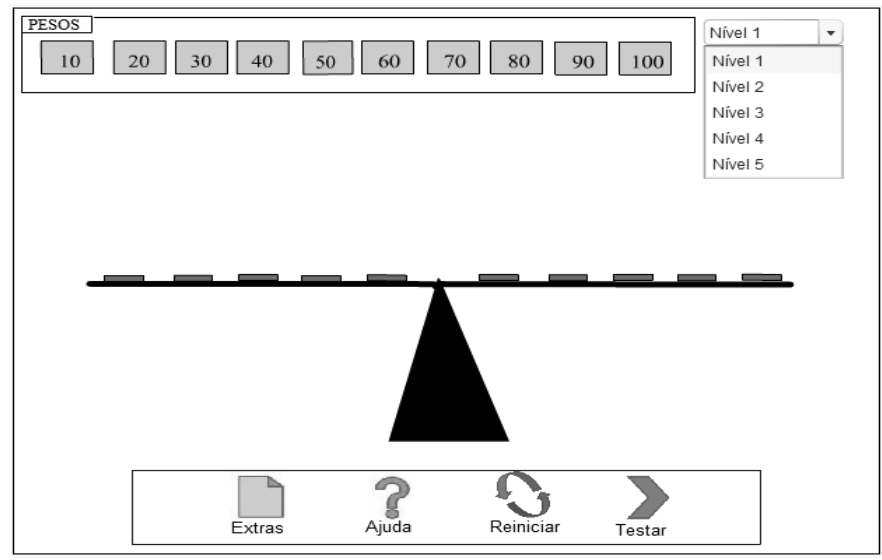

Figura 3 - Interface do OA Balança

Como estratégias para uso do OA envolvendo o papel e os recursos do QRCode, o professor pode utilizar:

- Problematização - A primeira estratégia seria a problematização. O professor pode utilizar o OA para iniciar o conteúdo sobre grandezas proporcionais, elaborar questões relacionadas ao OA e disponibilizá-las através do papel. Vale ressaltar a importância de considerar o conhecimento prévio do aluno sobre o assunto, para favorecer a aprendizagem significativa. O OA pode ser um recurso para aprimorar o conhecimento.

- Representação da solução - A segunda estratégia seria a representação da solução. De posse do papel com a interface do OA e das questões, o aluno pode refletir sobre a solução para o problema proposto e representar a solução no papel. Conforme abordam O'Hara e Sellen (1997) em uma comparação com ferramentas on-line, o papel oferece suporte a anotação enquanto leitura, navegação rápida e flexibilidade de layout espacial. Dessa forma, o aluno pode solucionar o problema e destacar, dentre as opções, aquela que julga resolver o problema proposto. Além da representação lógica (escrita no papel) usada na resolução do problema, pode ser solicitado ao aluno que ele justifique a sua resposta.

- Teste do desempenho - Por último, após a reflexão e elaboração da solução, o aluno com uma câmera de um dispositivo móvel (acesso ubíquo) pode direcioná-la para a etiqueta do QR-Code (relacionada à opção que julga correta) e verificar a resposta através da animação interativa do OA, recebendo feedback.

O OA fornece feedback ao aluno, nos casos de opção certa ou errada. Mas o objetivo é que o OA retorne um feedback apropriado a cada opção escolhida pelo aluno, 
com o objetivo de estimular a aprendizagem. Este tipo de feedback é essencial, pois o aluno, ao resolver um exercício em outro local, sem a presença do professor, poderá receber um feedback apropriado de forma imediata.

O professor também pode utilizar uma estratégia para um trabalho colaborativo com a formação de grupos, onde cada grupo possui um problema diferente a ser solucionado. Dessa forma, cada grupo apresentaria as tentativas e discussões realizadas pelos integrantes para obter a resolução do problema.

Através do papel o aluno consegue interagir com o OA usando um dispositivo móvel movimentando os pesos e colocando-os em diferentes distâncias. Ao escolher uma opção (representada por QR-Code), o OA executa a ação e exibe a animação correspondente. Se a balança equilibra-se, então o aluno conseguiu encontrar a solução para o problema proposto. Além de visualizar a animação correspondente a opção escolhida, o OA continua ativo permitindo que o aluno experimente outras possibilidades.

Conforme apresenta a Figura 4, o professor pode elaborar uma questão sobre grandezas usando o OA Balança. O aluno deve verificar que peso deve ser usado e em qual posição deve colocá-lo para equilibrar a balança. Para isso, ele deve saber os conceitos relacionados ao conteúdo de grandezas. Como mencionado acima, esse tipo de atividade pode ser usado como forma de trabalhar o conhecimento prévio para conteúdos mais complexos e que envolvam assuntos relacionados. 


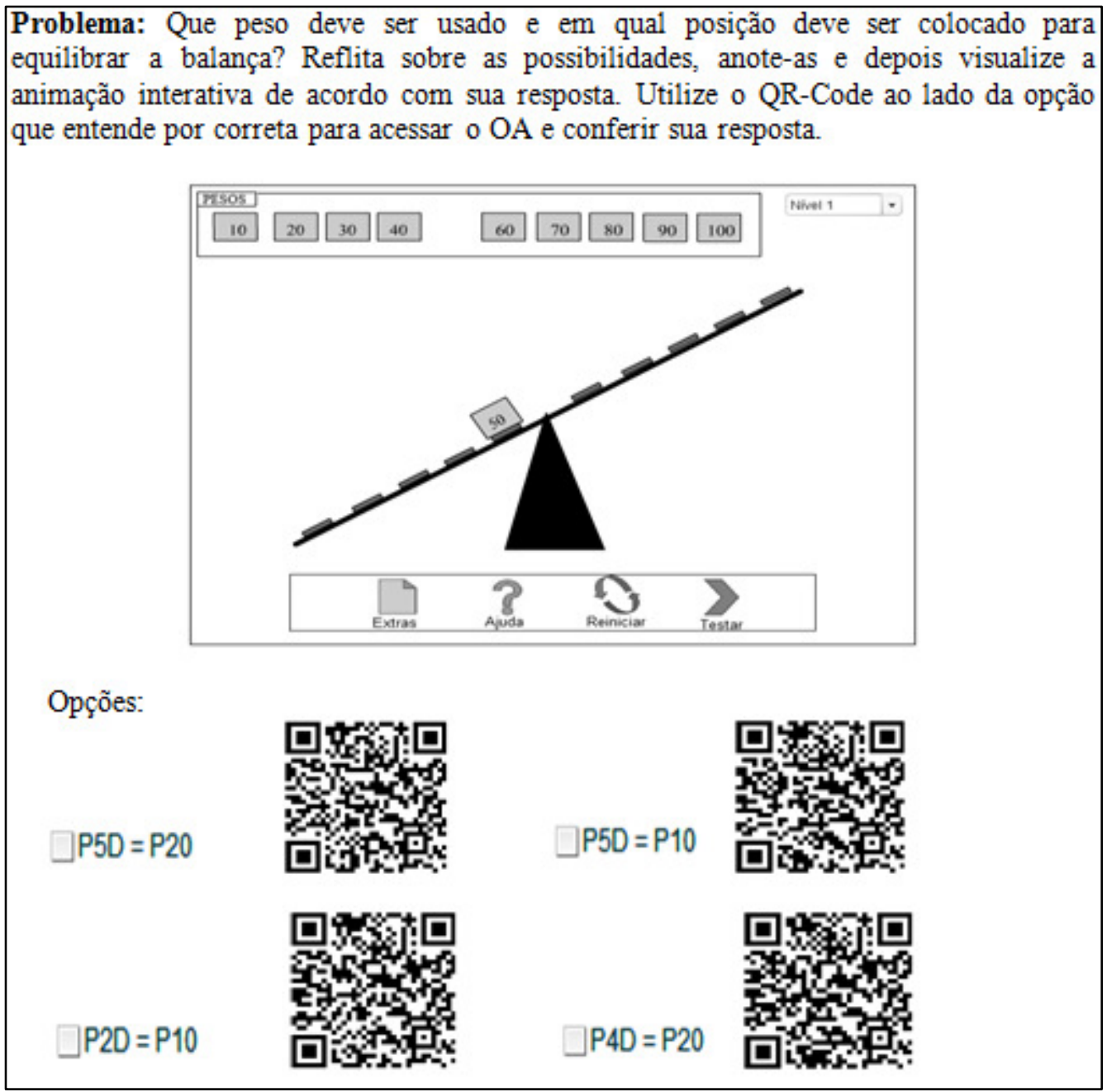

Figura 4 - Exemplo de um problema envolvendo o OA Balança

O OA impresso pode conter um QR-Code que o visualize por completo ou vários QR- Codes que redirecionem para a animação interativa do OA, correspondente a resposta do aluno. Se, para o problema da Figura 4, o aluno escolhe um QR-Code referente ao peso 10 (P10), na quinta posição do lado direito (P5D), então o aluno visualiza a animação que corresponde ao acerto (Figura 5). Para verificar o funcionamento da proposta do papel integrado a QR-Codes para acessar OA (Figura 5), foram utilizados o smartphones E63 e o N5230 (touchscreen), este último permitindo a manipulação de objetos, com o toque das mãos, diretamente na tela. 


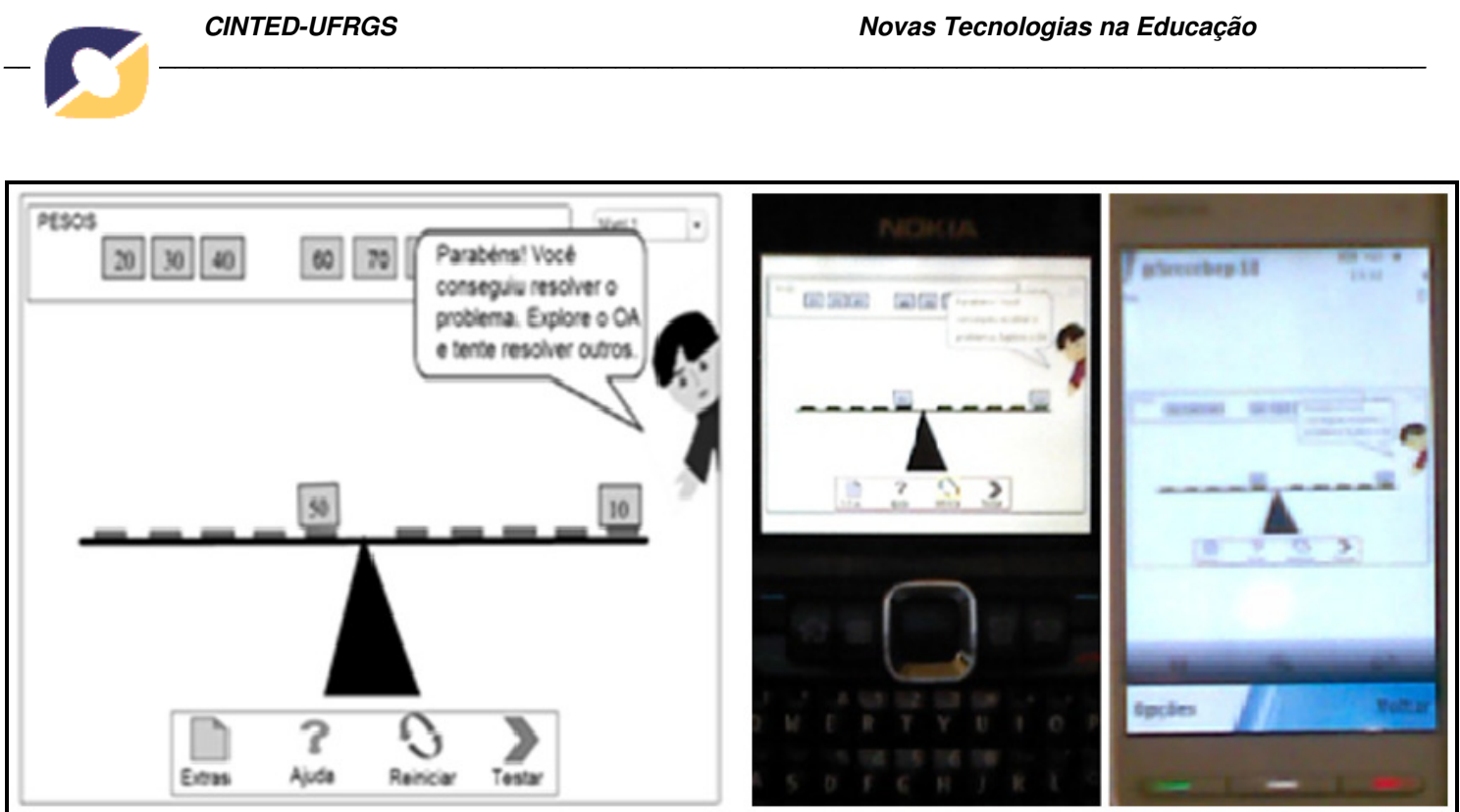

Figura 5 - Exemplo de retorno (na Web e nos dispositivos móveis, respectivamente) fornecido ao aluno após visualizar a opção escolhida

O ícone Testar na barra inferior permite testar o OA de acordo com as opções de peso e distância. O ícone reiniciar permite voltar a posição inicial do OA. O ícone Ajuda deve fornecer informações sobre o funcionamento do OA e como esse pode ser utilizado. Extras permite o acesso a informações extras relacionadas ao conteúdo educacional abordado pelo OA. Existe ainda a hipótese de elaborar um ícone para fornecer um feedback personalizado ao aluno. Por exemplo, se o aluno escolheu dentre algumas opções a que mais se difere da resposta correta, ele recebe um feedback diferente daquele na qual a resposta está mais próxima da opção correta.

Tal estratégia pode ser usada em sala de aula como uma estratégia para obter atenção do aluno, de forma ubíqua, onde o aluno pode acessar o OA a qualquer momento através do papel (com QR-Code) e um dispositivo móvel. Vale ressaltar a importância de estratégias diferentes em sala de aula, principalmente se considerarmos o público alvo formado por Homo Zappiens - incluem aqueles que cresceram usando múltiplos recursos tecnológicos desde a infância como o controle remoto, computador, o telefone celular, o iPod, o aparelho de mp3, entre outros (Veen e Vrakking, 2009).

A proposta do uso de mídia impressa integrada a QR-Code também pode ser utilizada como um recurso para a educação à distância. Belisário (2006) aborda que os cursos a distância têm se utilizado de material impresso, o que se caracteriza pela pouca interatividade, mas também aponta que o uso do computador, apesar da interatividade, também pode apresentar problemas como falta de hábito de leitura na tela, problema de acesso ao material e a necessidade de um ambiente adequado. Nesse caso, uma alternativa para este problema pode ser a proposta aqui apresentada, permitindo a ubiquidade e a interatividade. No caso da educação a distância, o aluno com o material impresso (contendo QR-Code) pode ter acesso a textos e imagens e ao utilizar um dispositivo móvel pode acessar o áudio, vídeo, texto, etc., relacionado ao mesmo conteúdo, através do QR-Code existente no papel. Para verificar o acesso ubíquo do aluno pretende-se disponibilizar o OA em um Ambiente Virtual de Aprendizagem com recursos que permitam o registro do acesso realizado pelo aluno. 


\section{4- Considerações finais}

Diante das possibilidades de acesso a conteúdos educacionais é possível elaborar estratégias que tornem as aulas mais interativas e motivadoras para os alunos, assim como possibilitar o acesso ubíquo a esses conteúdos. Este artigo apresentou uma proposta de uso do papel para acesso a OA como alternativa para proporcionar a ubiquidade, baseando-se na mobilidade que o papel e os dispositivos móveis proporcionam. A proposta será aplicada a alunos da $6^{\mathrm{a}}$ série para realização de prova de conceito e aperfeiçoamento das estratégias. Após a validação da proposta pretende-se aperfeiçoar o OA tornando-o genérico, ou seja, permitindo que todos os pesos e distâncias sejam representados por QR-Code. Além disso, a aplicação da proposta pode ser implantada em outros OA (outras áreas e conteúdos), que contenham vídeos, áudios, exercícios, etc. A proposta do uso do papel como meio para acesso a diversas mídias pode ser usada para facilitar o ensino a distância, uma vez que os conteúdos referenciados nos QR-Code podem ser atualizados na Web, gerando inclusive uma economia de material educacional, pois a fonte de informação pode continuar a mesma. Além disso, pode-se utilizar também o QR-Code como dispositivo de entrada para acessar conteúdos na TVD. Enfim, este artigo apresenta uma proposta inicial como alternativa para acesso ubíquo a conteúdos educacionais.

\section{Referências}

AL-KHALIFA, Hend, Utilizing QR-Code and Mobile Phones for Blinds and Visually Impaired People, In: K. Miesenberger et al. (Eds.), ICCHP 2008, LNCS 5105, pp. 1065-1069, 2008.

AUSUBEL, David, Aquisição e retenção de conhecimentos: uma perspectiva cognitiva. Lisboa: Plátano Edições Técnicas, 2003.

BARBOSA, Maria Lúcia Kroeff; ROESLER, Valter; REATEGUI, Eliseo, Uma proposta de modelo de interface interoperável para web, TV digital e dispositivos móveis, RENOTE- Novas Tecnologias na Educação, V. 7 № 1, Julho, 2009.

BELISÁRIO, Aluízio, O material didático na educação a distancia e a constituição de propostas interativas, In: SILVA, M. (org), Educação Online, São Paulo:Loyola, 2006. p. 137-148.

FURLAN, Rogério; EHRENBERG, Karla Caldas, Desenvolvimento de conteúdo audiovisual para dispositivos móveis, In: SQUIRRA, S.; BECKER, V. (orgs), TVDigital.br, São Paulo:Ateliê Editorial , p.171 -189, 2009.

GHIRON, Stefano Levialdi; MEDAGLIA, Carlo Maria; PERRONE, Amedeo, "Artsonomy": Social Bookmarking of Real Artworks via Mobile Applications with Visual Tags, Part III, HCII 2009, LNCS 5616, pp. 375-384, 2009.

GOMES, Fábio Jesus Lima, Explorando Objetos de Aprendizagem na TV digital: estudo de caso de alternativas de interação, no Estado do Rio Grande do Sul. Porto Alegre:PGIE/UFRGS, 2009. 162p. Tese de Doutorado.

HEATH, Christian; LUFF, Paul, Technology in Action. Cambridge: Cambridge University Press, 2000. 
IEEE, L.T.S.C.; IEEE Standard for learning object medadata, 2002. Disponível em:< http://ltsc.ieee.org/wg12/index.html> Acesso em 28 Set. 2010

KLEMMER, Scott et al., Books with Voices: Paper Transcripts as a Tangible Interface to Oral Histories, CHI 2003: NEW HORIZONS, Ft. Lauderdale, Florida, USA p.89-96, 2003.

LYTRAS, Miltiadis et al., A. Interactive Television and e-Learning Convergence: Examining the Potential of t-Learning. European Conference on e-Learning, 11 p., 2002.

MELO, Bergson Rodrigo Siqueira; VASCONCELOS, Francisco Herbert Lima.; PEQUENO, Mauro Cavalcante; FILHO, Jose Aires Castro e SILVA, Veronica Maria Lavor, Objeto de Aprendizagem Gangorra Interativa na Compreensão Conceitual de Grandezas. WIE - Workshop sobre Informática na Escola, Belém-PA. 2008

MIYAOKU, Kento; TANG, Anthony; FELS, Sidney, C-Band: A Flexible Ring Tag System for Camera-Based User Interface, In: R. Shumaker (Ed.), Virtual Reality, HCII 2007, LNCS 4563, pp. 320-328, 2007.

O'HARA, Kenton; SELLEN, Abigail, A Comparison of Reading Paper and On-Line Documents. In Proceedings of CHI 97, USA, p. 335-342. ACM Press. 1997.

O'MALLEIY, C. et al., WP4-Guidelines for learning/teaching/tutoring in a mobile environment, MOBIlearn, $2005 . \quad$ Disponível em: $<$ http://www.mobilearn.org/download/results/guidelines.pdf> Acesso em 20 Set. 2010

PELLANDA, Eduardo Campos, Comunicação móvel no contexto brasileiro, In: LEMOS, A, JOSGRILBERG, F. (orgs), Comunicação e mobilidade- aspectos socioculturais das tecnologias móveis de comunicação no Brasil, Salvador: EDUFBA, 2009. p. 11-18.

RAMSDEN, Andy, The use of QR-Codes in Education: A getting started guide for academics. Online Publications Store, University of Bath Opus, 2008.Disponível em: < http://opus.bath.ac.uk/> Acesso em 15 Set. 2010.

SELLEN, Abigail; HARPER, Richard, The Myth of the Paperless Office. Cambridge, MA: MIT Press, 2001.

TANAKA, Michiru; TESHIGAWARA, Yoshimi, A Method and Its Usability for User Authentication by Utilizing a Matrix Code Reader on Mobile Phones, In: J.K. Lee, O. Yi, and M. Yung (Eds.), WISA 2006, LNCS 4298, pp. 225-236, 2007.

VEEN, Wim; VRAKKING, Ben, Homo Zappiens educando na era digital, Porto Alegre: Artmed, 2009.

VERNE, Jules, Around the world in 80 days -The ubitour version guided by your mobile phone, Indianapolis : Ubimark Books, v. 1, 2010

YOSHIDA, Yuichi; MIYAOKU, Kento; SATOU, Takashi, Mobile Magic Hand:Camera Phone Based Interaction Using Visual Code and Optical Flow, J. Jacko (Ed.), Human-Computer Interaction, Part II, HCII 2007, LNCS 4551, pp. 513-521, 2007. 